Accelerator Division

Alternating Gradient Synchrotron Department

BROOKHAVEN NATIONAL LABORATORY

Upton, New York 11973

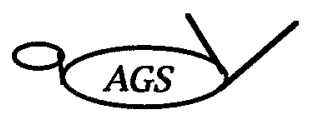

Accelerator Division

Technical Note

AGS/AD/Tech Note No. 447

\title{
MAD Simulation: HEP/SEB Extraction (FY1996)
}

M. (Sanki) Tanaka

11 October 1996

Version 1.1

(Ver. 1.1: Fixed an error on Dx in Fig.4.5 and Table 3.)

(Ver. $1.0: 10$. Oct.96)

\begin{abstract}
The slow extracted beam (SEB) at the AGS has been used successfully since1968 for the the High Energy Physics Program. This note desicribes the results from a MAD tracking study of SEB resonant extraction up to straight section F13 using the actual FY96 HEP/SEB run parameters and an estimated emittance for the high intensity beam of (5-6) $10^{13} \mathrm{ppp}$.
\end{abstract}




\section{Introduction}

The slow extracted beam (SEB) from the AGS to the East Area has been operational since March 1968 for the High Energy Physics Program (HEP)[1]. The present SEB system with an electrostatic wire septum at straight section H20 was implemented in 1979-1980[2] together with the new SEB switchyard[3]. In the SEB mode the debunched circulating beam is extracted to over 1 to $2 \mathrm{sec}$ (spill length) for the typical repetition time of 2.4 to $4.0 \mathrm{sec}$ by exciting a third integer resonance $\left(3 \cdot \mathrm{Qh}_{\mathrm{h}}=26\right)$. The long and uniform spill allows HEP counter experiments to take data smoothly without overloading their detectors. For typically good SEB operation during the pre-Booster HEP program, the measured extraction inefficiency was 3-5 \% for the extracted beam intensity of 1.0-1.6 $10^{13} \mathrm{ppp}$ at $\mathrm{p}=25-29 \mathrm{GeV} / \mathrm{c}[4,5]$. In FY1995-6 HEP/SEB runs, we have had a 1-3\% inefficiency for the record high intensity of $5.0-6.0 \cdot 10^{13} \mathrm{ppp}$ at $\mathrm{p}=$ 25.5 GeV/c according to the AGS Daily Report (see Fig. 1). This SEB performance looks great if it is the case. However, the unexpected large beam losses were observed in the Switchyard, indicating that the extracted beam emittance might have become too large. This note describes the results from a MAD tracking study of AGS SEB extraction up to the middle of straight section F13 (ssF13md) using the FY96 HEP/SEB run parameters and an estimated high intensity beam emittance, hoping they might shed some light on the problem.

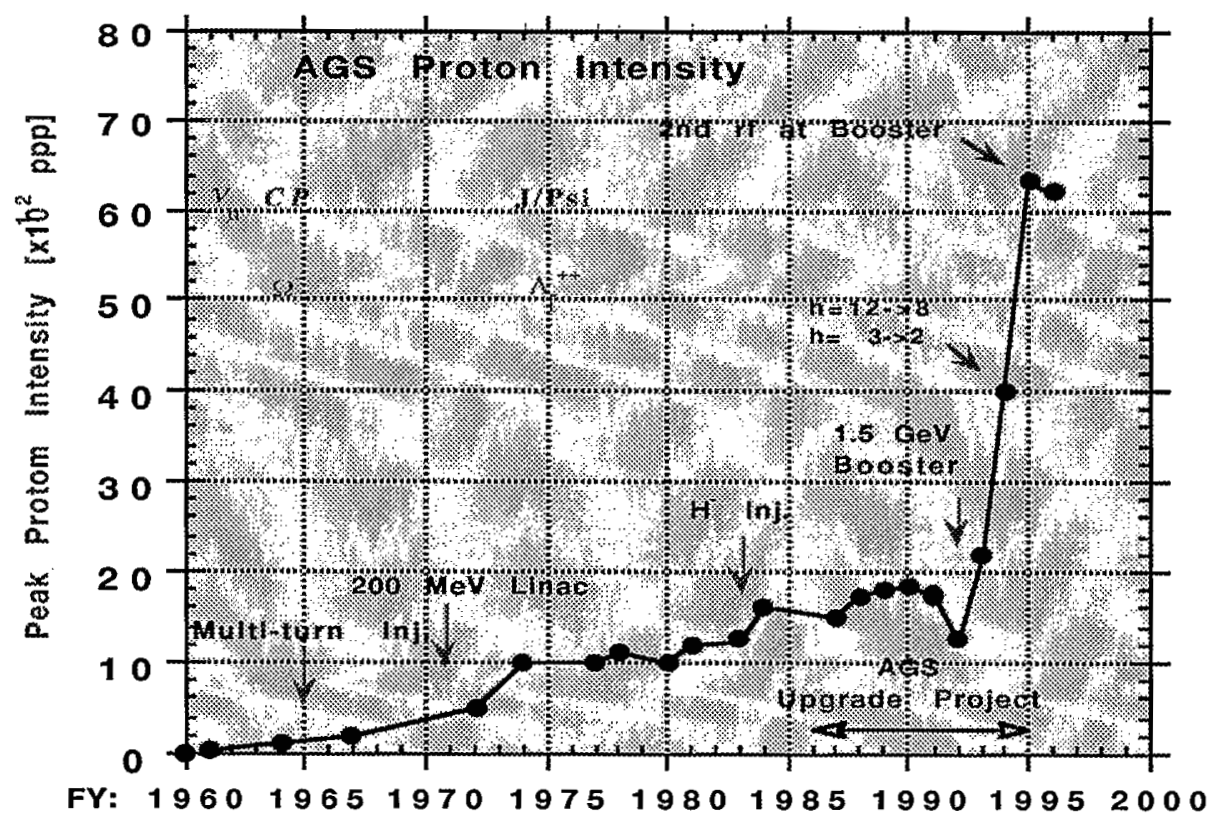

Fig. 1. AGS Proton Intensity Performance from 1960 to 1996. 


\section{SEB Extraction}

\subsection{SEB Extraction System}

The present SEB system consists of an electrostatic wire septum at sssH20 [ESH20], a thin septum magnet at F05 [SMF05], and a thick ejector magnet at F10 [SMF10], together with two $3 / 2 \lambda$ local orbit deformations [HPBLW, FPBLW] and 4 drive sextupoles [DS] which excite the third-integer resonance $[2,4]$. The layout of the main SEB extraction elements in the ring are schematically shown in Fig. 2.

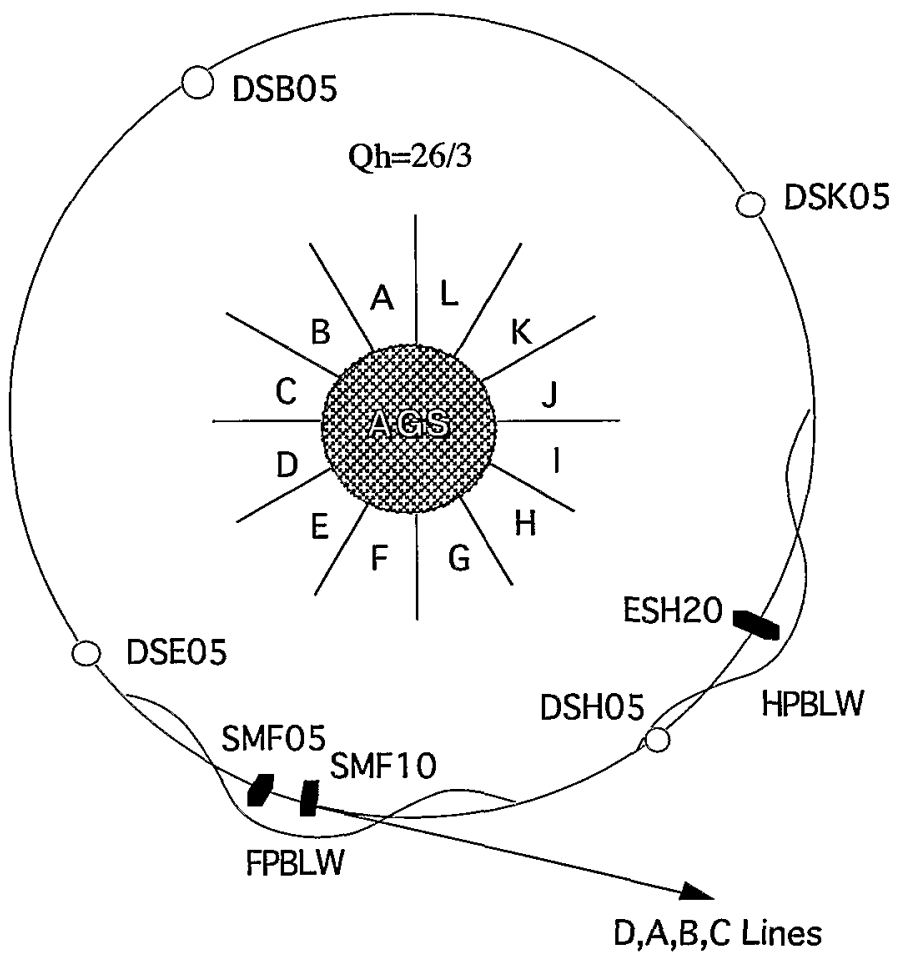

Fig. 2. The layout of the SEB extracion elements.

\subsection{Third-integer Resonant Extraction Principle}

AGS SEB extraction is performed by controlled excitation of nonlinear third integer betatron resonance of the main ring at $3 \cdot \mathrm{Qh}=26$, which corresponds to the tune value $\mathrm{Qh}_{\mathrm{h}}$ of the unperturbed ring near the center of the vacuum chamber. The factor " 3 " arises from the drive sextupole fields which excite the resonance and the integer 26 from the 26-th Fourier harmonic component of the fields. For each momentum, there is a set of three linear separatrices which define a stable horizontal phase space area with a triangle shape. The stable triangular area can be reduced by adjusting the tune to be in the vicinity of $\mathrm{Qh}=26 / 3$ either directly by varying the tune quadrupole strength or indirectly through slow beam steering or by increasing the drive sextupole strength. At the AGS, the tune quadrupole and drive sextupole strengths are kept constant during extraction, and the beam is pushed slowly from its stable position to the resonance by a slightly negative main magnet flattop slope so that different momentum particles progressively move on to the resonance. Extraction starts when the beam emittance fills the 
stable area for the higher end of momentum distribution. As the stable area slowly shrinks to zero for these particles, other parts of the momentum distribution are subsequently extracted.

The extraction inefficiency depends mainly on the fraction of particles which strike the septum of the first extraction device (ESH20) and is given by ineff. $=f \cdot w / s$, where $w$ is the effective septum thickness, "s" is the step size (spiral pitch, the growth of the resonant betatron amplitudes in the final few turns before extraction) and $\mathrm{f}$ is a factor $(\sim 1.5)$ to account for the horizontal beam density variation. The step size $\mathrm{s}$ of a particle depends on its momentum, initial amplitude and its proximity to the resonant tune value. The maximum value of the step size is limited by the available aperture at ESH20, downstream and the circulating beam emittance. For the analytical aspect of SEB extraction, see ref. [1].

\subsection{SEB Extraction Process at AGS}

Once the beam is accelerated to the $25.5 \mathrm{GeV} / \mathrm{c}$ flattop, the if is turned off and the beam is debunched and the the momentum spread is increased. Extraction devices ESH20, SMF05 and SMF10 as well as high field quadrupoles are turned on before rf turn-off, and the 4 Drive Sextupoles [DS], Backleg Windings [FPBLW ,HPBLW] are energized shortly after the rf turn-off.

The equilibrium orbit of the beam at this time is adjusted to be located at approximately $8.0 \mathrm{~mm}$ inside of the resonance radius $\left(R_{0}=0\right)$ and $Q_{h}=\sim 8.75$. Since the horizontal chromaticity is negative, the beam spirals outward to the radius near the center corresponding to the $\mathrm{Qh}=8^{2} / 3$ resonance by slowly decreasing the the flottop fields in the AGS main magnets. As the beam tune approaches the resonance, the stable region (separatrix) in the horizontal phase plane shrinks for each momentum. For each momentum, there is a set of three separatrices which forms a stable triangular area. The lower the momentum, the larger the stable triangular area is.

Extraction starts when the separatrix area for the highest momentum particles are equal to the phase plane area enclosed by the orbit of the particles of that momentum with the highest betatron oscillation amplitude. Slightly later, particles of a higher momentum with smaller amplitudes will be extracted simultaneously with the particles of a lower momentum with larger amplitudes. Particles outside the separatrix execute oscillations of rapidly increasing amplitude and move outward each of three asymptotes associated with the third integer resonance separatrix. For every three turns the change of the particle position (or step size) increases rapidly as it approaches the septum of ESH20 in the phase space. The particle eventually jumps across the wire septum and is kicked outward. After deflection by ESH20, the particle turns around about $2^{3 / 4}$ orbits and then arrives at SMF05 in the proper phase for further deflection by this septum. At the distance $=24$ full betatron oscillations, the angular deflection $\mathrm{dx}^{\prime}$ at ESH20 will, in the linear case, produce no net spatial separation at SMF05 since $\mathrm{dx}(\mathrm{F} 5)=$ $\mathrm{dx}^{\prime} \cdot \sqrt{ }[\mathrm{B}(\mathrm{H} 20) B(\mathrm{~F} 5)] \cdot \sin (\Delta \mu)=\sim 0$, where $\Delta \mu=$ the phase advance between $\mathrm{H} 20$ and $\mathrm{F} 5=\sim 24 \cdot 2 \cdot \pi$. 
However, the non-linear effects of the sextupole moments of the drive sextupoles and of the main magnets are significant enough to produce the required displacement at SMF05 The beam kicked by SMF05 is finally extracted to the external beam line by the ejector magnet SMF10. A comprehinsive description of the AGS SEB extraction system is given in ref.[4].

\subsection{SEB Extraction Setup Parameters}

During the FY95/96 HEP/SEB runs, the typical machine setup paramters on the SEB flattop were

$\begin{array}{lll}\cdot \text { Main Magnet } & 4484 \mathrm{~A} & ! 1.0052 \mathrm{~T} \text { or } \mathrm{p}=25.725 \mathrm{GeV} / \mathrm{c} \\ \cdot \text { HBLW } & \sim 267 \mathrm{~A} & \\ \cdot \text { FBLW } & \sim 297 \mathrm{~A} & \\ \cdot \text { DS } & \sim 300 \mathrm{~A} \\ \cdot \text { ESH20 } & \sim 50 \mathrm{kV} & \\ \cdot \text { SMF05 } & \sim 2.2 \mathrm{kA} & \\ \cdot \text { SMH10 } & \sim 4.4 \mathrm{kA} & \end{array}$

While there are no recent realiable measurements of the transverse emittance of the circulating high intensity proton beam in the AGS, the consensus is that the $95 \%$ normalized emittance should be $\varepsilon^{*} \mathrm{~h}, \mathrm{v}(95 \%)=(75-95) \pi \mathrm{mm}-\mathrm{mrad}$, significantly higher than the pre-booster values $\varepsilon^{*} \mathrm{~h}, \mathrm{v}(95 \%)=$ $6 \cdot \sigma^{2} / B \cdot(\mathrm{p} / \mathrm{m})=(25-40) \pi \mathrm{mm}$-mrad. During the FY96 HEP/SEB run, the chromaticity is corrected to be $\left\{\xi_{\mathrm{h}}, \xi_{\mathrm{v}}\right\}=\{-1.8,-0.22\}$ with $\left.\left\{\mathrm{I}_{\mathrm{Sh}}, \mathrm{I}_{\mathrm{Sv}}\right\}=150,70\right\} \mathrm{A}$ and $\left(\mathrm{Q}_{\mathrm{h}}, \mathrm{Q}_{\mathrm{v}}\right\}=\sim\{8.67,8.78\}$ with $\left\{\mathrm{I}_{\mathrm{Qh}}, \mathrm{I}_{\mathrm{Qv}}\right\}=$ $\sim\{80,-160\} \mathrm{A}$ on the flattop at $\mathrm{Ro}=0$ to cope with the obseved vertical beam instability. Table 1 summarizes some basic parameters of ESH20, SMF05 and SMF10.

Table 1. Parameters of the SEB extraction devices

\begin{tabular}{|l|lll|l|}
\hline & ESH20 & SMF05 & SMF10 & \\
\hline gap(v) x width(h) & $20 \times 10$ & $31.75 \times 17.5$ & $60.0 \times 25.9$ & mm x mm \\
length & 2.30 & 0.667 & 2.06 & $\mathrm{~m}$ \\
$\mathrm{I}_{\max }$ & $(80 \mathrm{kV})$ & 2.2 & 5.0 & $\mathrm{kA}$ \\
$\mathrm{B}_{\max }$ & $(80 \mathrm{kV} / \mathrm{cm})$ & 0.15 & 0.97 & $\mathrm{~T}$ \\
Septum width & $0.051(\mathrm{~W} / \mathrm{Re})$ & $0.76(\mathrm{Cu})$ & $15.9(\mathrm{Cu})$ & $\mathrm{mm}$ \\
Kick(nominal) & 0.43 & 1.1 & 18.5 & $\mathrm{mrad}$ \\
\hline
\end{tabular}




\section{MAD Simulation}

The simulation of the AGS SEB extraction was made by using the BNL MAD code[6] including the following elements:

-Main Combined Function Magnets containing mutipoles up to sextupoles,

-High Field Tune Quadrulpoles,

-Chromaticity Sextupoles,

-SEB Extraction devices (HPBLW, FPBLW, DSs, ESH20, SMF05, SMF10).

MAD was run to obtain the working point parameters and extraction closed orbit deformations using the actual FY96 HEP/SEB run parameters. Results are shown in MAD FTWISS output format in Table2 and in Fig. 3.

Table 2. FTWISS output for the SEB working point $(\mathrm{p}=25.5 \mathrm{GeV} / \mathrm{c}$ and $\mathrm{dp} / \mathrm{p}=0.0068$ at IDS $=300$ $\mathrm{A},\left\{\mathrm{I}_{\mathrm{Qh}}, \mathrm{I}_{\mathrm{Qv}}\right\}=\{80,-160\} \mathrm{A},\left\{\mathrm{I}_{\mathrm{Sh}}, \mathrm{I}_{\mathrm{Sv}}\right\}=\{150,70\} \mathrm{A}, \mathrm{I}_{\mathrm{HPBLW}}=270 \mathrm{~A}$ and $\left.\mathrm{I}_{\mathrm{FPBLW}}=300 \mathrm{~A}\right)$.

\begin{tabular}{|c|c|c|c|c|c|c|c|c|c|c|c|c|}
\hline ELEMENT SEQ & & & $\mathrm{HO}$ & R IZ & ONT & A L & & & & VEI & $\mathrm{R}$ T I C & A L \\
\hline POS. ELE & I & DIST & $\beta_{h}$ & $\alpha_{\mathrm{h}}$ & $\mu_{\mathrm{h}}$ & $\mathrm{x}$ & $x^{\prime}$ & $D_{x}$ & $\mathrm{D}_{\mathrm{X}}{ }^{\prime} \mathrm{I}$ & $\beta_{\mathrm{V}}$ & $\alpha_{\mathrm{V}}$ & $\begin{array}{l}\mu_{\mathrm{V}} \\
\text { [2PI] }\end{array}$ \\
\hline BEGIN AGS & 1 & 0.000 & 21.750 & -1.790 & 0.000 & 37.255 & 2.465 & 1.695 & 0.118 & 11.575 & 1.037 & 0.000 \\
\hline 138 DSK05 & 1 & 150.529 & 27.328 & 0.101 & 1.592 & 0.400 & 0.059 & 2.245 & -0.014 & 9.404 & -0.068 & 1.658 \\
\hline 313 DSB05 & 1 & 352.298 & 18.550 & 0.044 & 3.770 & 2.155 & 0.068 & 1.811 & -0.014 & 10.816 & -0.012 & 3.846 \\
\hline 488 DSE05 & 1 & 554.067 & 24.802 & -0.304 & 45.967 & 3.096 & 0.008 & 1.659 & 0.008 & 9.367 & 0.064 & 6.035 \\
\hline 551 SMF05us & 1 & 620.666 & 23.970 & 0.161 & 6.648 & 39.367 & 1.040 & $1.922-$ & -0.016 & 10.206 & 0.016 & 6.767 \\
\hline 552 SMF05ds & 1 & 621.326 & 23.776 & 0.133 & 6.652 & 40.053 & 1.040 & 1.911 & -0.016 & 10.229 & -0.049 & 6.777 \\
\hline 569 SMF10us & 1 & 636.795 & 15.940 & 1.258 & 6.824 & 37.497 & -4.030 & 1.656 & -0.115 & 13.301 & -1.149 & 6.948 \\
\hline 570 SMF $10 \mathrm{md}$ & 1 & 637.810 & 13.555 & 1.094 & 6.835 & 33.410 & -4.030 & 1.544 & -0.115 & 15.812 & -1.326 & 6.959 \\
\hline 571 SMF10ds & 1 & 638.825 & 11.502 & 0.929 & 6.848 & 29.322 & -4.030 & 1.432 & -0.115 & 18.683 & -1.503 & 6.969 \\
\hline $580 \mathrm{ssF} 13 \mathrm{md}$ & 1 & 648.043 & 23.514 & -0.083 & 36.957 & 15.375 & -1.919 & 1.965 & 0.007 & 10.058 & -0.020 & 7.064 \\
\hline 690 DSH05 & 1 & 755.836 & 24.085 & -0.107 & 78.112 & -18.121 & -0.087 & 2.330 & 0.019 & 9.960 & -0.003 & 8.236 \\
\hline 742 ESH20us & 1 & 804.826 & 14.677 & -1.356 & 58.646 & 31.714 & 2.465 & 1.435 & 0.118 & 17.148 & 1.441 & 8.759 \\
\hline 743 ESH20ds & 1 & 807.076 & 21.750 & -1.790 & 8.667 & 37.255 & 2.465 & 1.695 & 0.118 & 11.575 & 1.038 & 8.785 \\
\hline END AGS & 1 & 807.076 & 21.750 & -1.790 & 08.667 & 37.255 & 52.465 & 1.695 & 0.118 & 11.575 & 1.038 & 8.785 \\
\hline $\begin{array}{l}\mathrm{C}=2 \pi \mathrm{R}= \\
\Delta \mathrm{C}=8.32398\end{array}$ & & $\begin{array}{l}7.075641 \\
\mathrm{~mm}\end{array}$ & $\mathrm{~m}$ & $\begin{array}{l}\mathrm{Qh}= \\
\mathrm{Qh}^{\prime}=\end{array}$ & $\begin{array}{r}8.666 \\
-15.424\end{array}$ & $\begin{array}{l}550 \\
4595\end{array}$ & & $\mathrm{~V}^{\prime}=$ & $\begin{array}{l}3.78491 \\
1.95325\end{array}$ & & & \\
\hline$\alpha=0.1413$ & 48 & -01 & $B_{h}{ }^{n}$ & $\max =$ & 29.304 & $4740 \mathrm{~m}$ & $B_{v} \mathrm{ma}$ & & 4.9308 & $12 \mathrm{~m}$ & & \\
\hline$\gamma_{\mathrm{tr}}=8.41115$ & & & & $\begin{array}{l}\max = \\
\max =\end{array}$ & $\begin{array}{l}=2.66 \\
=43.60\end{array}$ & $\begin{array}{l}4284 \mathrm{~m} \\
7278 \mathrm{~m}\end{array}$ & $\mathrm{D}_{\mathrm{y}}^{\mathrm{ma}}$ & $a x=0$ & 0.00000 & & & \\
\hline
\end{tabular}

$\dagger \mathrm{Q}^{\prime}=\xi \cdot \mathrm{Q}=\mathrm{dQ} /(\mathrm{dp} / \mathrm{p})$ 

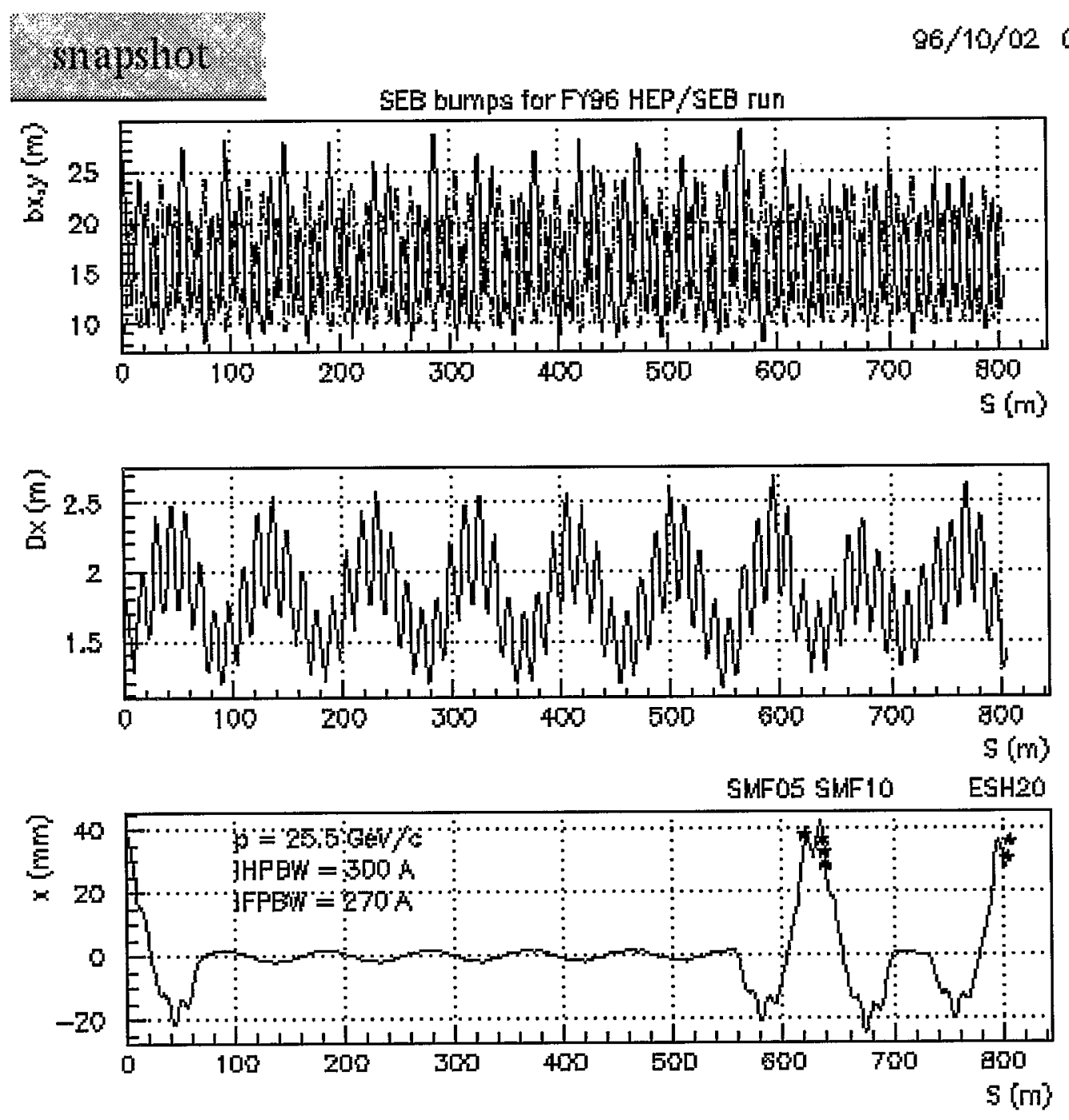

Fig. 3. $B_{h, v}, D_{X}$ and $x$ (SEB bumps) as a fuction of $S$ starting fromESH20ds at the SEB working point. 


\subsection{Tracking of the Resonance}

Particle tracking in the horizontal plane has been performed using MAD with an interactive graphical display utility[6]. During the extraction process, the stability of particles depends on both their momentum $\mathrm{dp} / \mathrm{p}$ and betatron amplitude $\left(\mathrm{x}, \mathrm{x}^{\prime}\right)$. The $\mathrm{dp} / \mathrm{p}$ values corresponding to the zero and nominal emittance of the circulating beam have been obtained by trying as close as possible to the resonance for the zero emittance case, and by trying out various sets of initial values for the nominal emittance case. Tracking was performed for each case, varying the initial values in small steps until two adjacent trajectories corresponding to the largest stable and the smallest unstable emittance were obtained as illustrated in the Fig. 4.0.

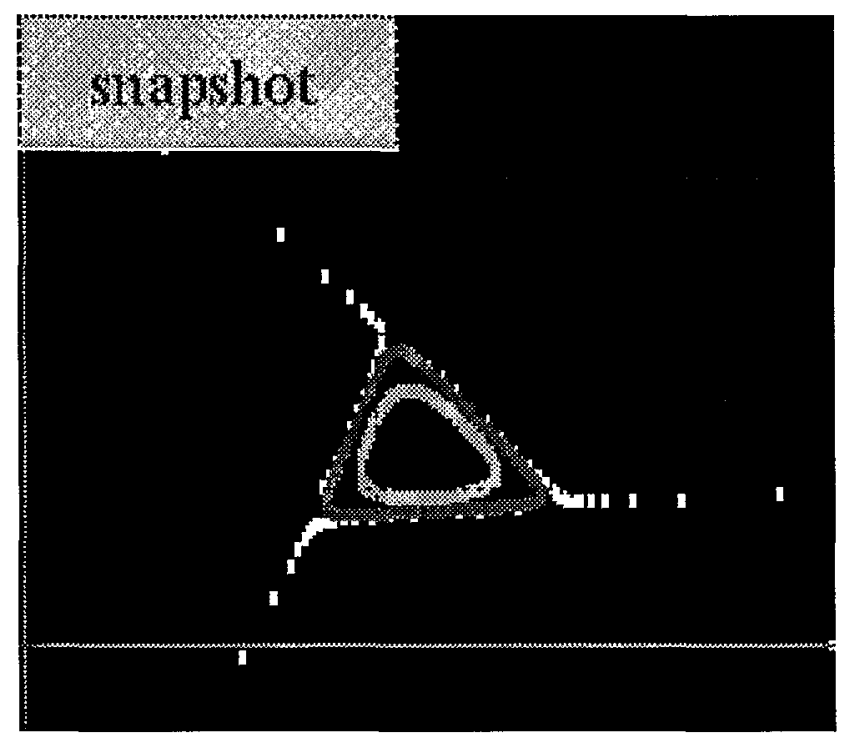

Fig. 4.0. Phase space $\left(x, x^{\prime}\right)$ output at SMF05 from tracking particles from various initial conditions for the fixed $\mathrm{dp} / \mathrm{p}$.

The nominal emittance of the circulating beam is estimated by calculating an area of the largest stable triangle. The tracking results show that the $\mathrm{dp} / \mathrm{p}$ value for the zero emittance and for the nominal emittance $\left(\varepsilon_{\mathrm{h}}=\sim 3.3 \pi \mathrm{mm}\right.$-mrad which is corresponding to $\left.\varepsilon^{*} \mathrm{~h}=\sim 90 \pi\right)$ are $\mathrm{dp} / \mathrm{p}=0.000650 \mathrm{and} \mathrm{dp} / \mathrm{p}=$ $=0.0000785$, respectively. Tracking the unstable particles is used to calculate the step size at ESH20. Tracking was stopped once the particle had jumped the first wire septum. The positions of the successive turns are interpolated along the extraction separatrix to find the value of $x$ ' at the septum position and the next $\left(x, x^{\prime}\right)$ values after 3 turns. Fig. 4.1 shows the results at the beginning of ESH20 and also at the end with a septum kick of $0.35 \mathrm{mrad}$. The step size is calculated to be $\sim 7.5 \mathrm{~mm}$ for the zero emittance case and $\sim 3.7 \mathrm{~mm}$ for the nominal one at $\mathrm{x}_{\mathrm{sep}}=47.0 \mathrm{~mm} \dagger$, and the instantaneous external beam emittance (an

$\dagger$ Practically all the beam loss occurs at the upstream end of the septum. The setp size is $(4.6-9.5) \mathrm{mm}$ at the end of the septum. 
area of the trapezoid) is $\sim 0.60 \pi \mathrm{mm}$-mrad. Tracking continues with starting values ( $\mathrm{x}, \mathrm{x}^{\prime}$ ) at four points which define the external beam emittance. These four particles are traced through the extraction channel and receive 0.35 mrad kick at ESH20, 1.45 mrad kick at SMF05 and $19.0 \mathrm{mrad}$ kick at SMF10 up to the middle of ssF13, where the extracted beam should be $\sim 380 \mathrm{~mm}$ away from the central orbit, free from the fringe field of the ring magnets.

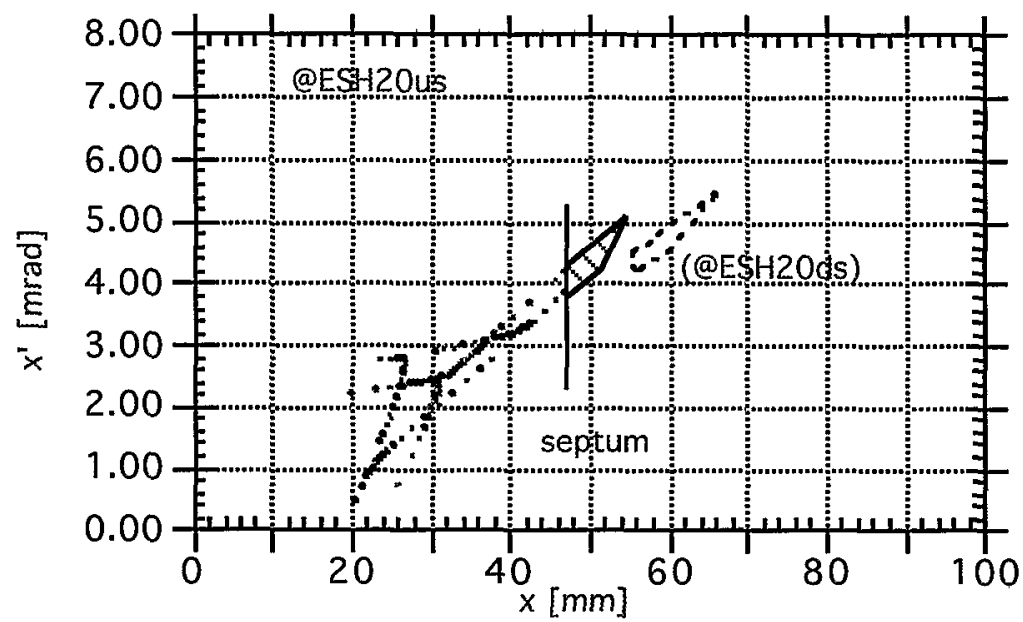

Fig. 4.1. Phase space $\left(x, x^{\prime}\right)$ at the beginning of ESH20.

\subsection{Tracking of the Extracted Beam}

From the first septum (ESH20) to the second septum (SMF05), tracking is done for $2^{3 / 4}$ turns for particles that jumped the wire septum and were kicked outward by $0.34 \mathrm{mrad}$ by ESH20. The clearance between the circulating beam and the extracted one at SMF05us is $\sim 1.14 \mathrm{~mm}$, marginal but acceptable as the SMF05 septum thickness is $0.9 \mathrm{~mm}$. The step size is $(9.5-31) \mathrm{mm}$ so that the beam could fill up the available horizontal aperture of $32 \mathrm{~mm}$ at SMF05. The external beam emittance $\varepsilon_{\mathrm{h}}{ }^{\text {ext }}$ is calculated to be $\sim 1.8 \pi \mathrm{mm}$-mrad, increased from $\sim 0.60 \pi \mathrm{mm}$-mrad at ESH20. The results are shown in Fig. 4.2 with the beam kicked further outward 1.46 mrad by SMF05. The particles kicked by SMF05 are then traced though the third septum SMF10 The clearance at SMF10us is $23.1 \mathrm{~mm}$, quite adequate for the $16 \mathrm{~mm}$ thick septum as seen Fig. 4.3. The external emittance is calculated to be $\sim 2.0 \pi \mathrm{mm}$-mrad at this point, which corresponds to $\varepsilon^{*}{ }_{h}^{\text {ext }}=\sim 54 \pi \mathrm{mm}$-mrad, $60 \%$ of the internal emittance of $90 \pi \mathrm{mm}$-mrad.. 


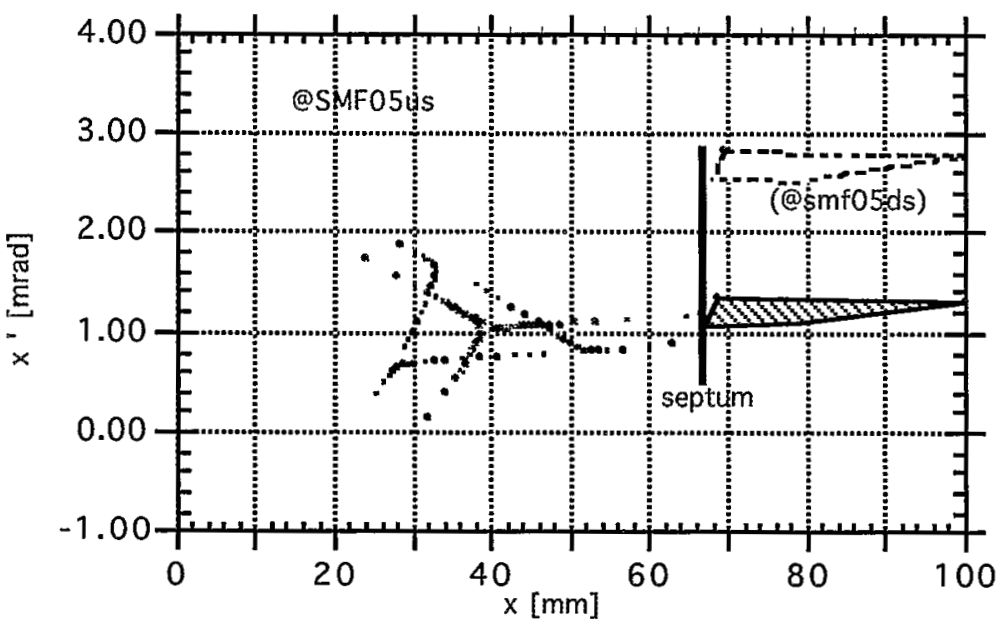

Fig. 4.2. Phase space at the beginning of SMF05.

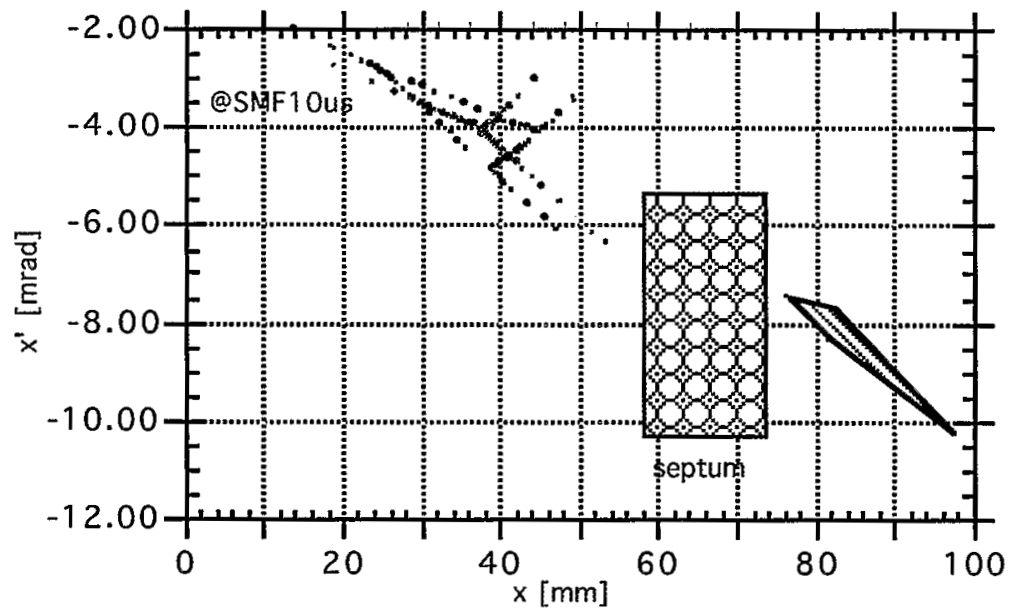

Fig. 4.3. Phase space at the beginning of SMF10.

The beam extracted by 19 mrad kick at SMF10 will cross the stray field of the two horizontally defocusing AGS main magnets (F11 and F12) and pass through the fringe field of the F13 main magnet at $x>250 \mathrm{~mm}$, where its gradient reverse sign and becomes defocusing. Since the standard AGS model uses the field parameterization which is good up to $\pm 100 \mathrm{~mm}$, we built a simple model of parameterizations of F11, F12 and F13 magnet based on the values calculated by the POISSON code and the knowledge of where the beam must pass though at each magnet. Fig. 4.4 shows the phase space plot at ssF13md. The external emittance has not changed and is $\sim 2.0 \pi \mathrm{mm}$-mrad. Fig. 4.5 and Table 3 show the Twiss parameters and beam positions as a fuction of $\mathrm{S}$ from SMF05ds to ssF13md. 


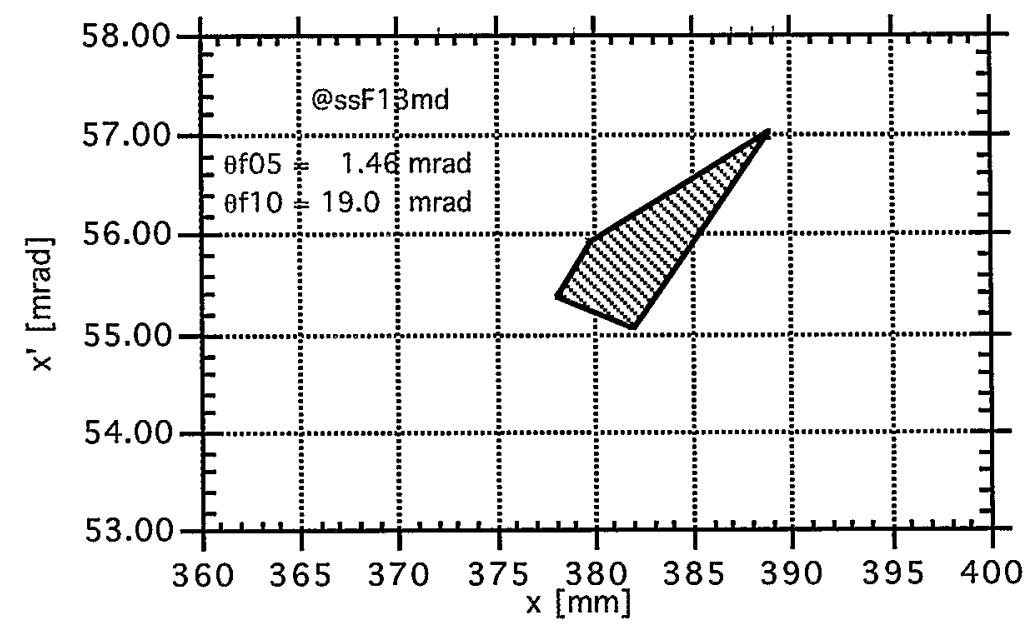

Fig. 4.4. Phase space at $\mathrm{ssF} 13 \mathrm{md}$.
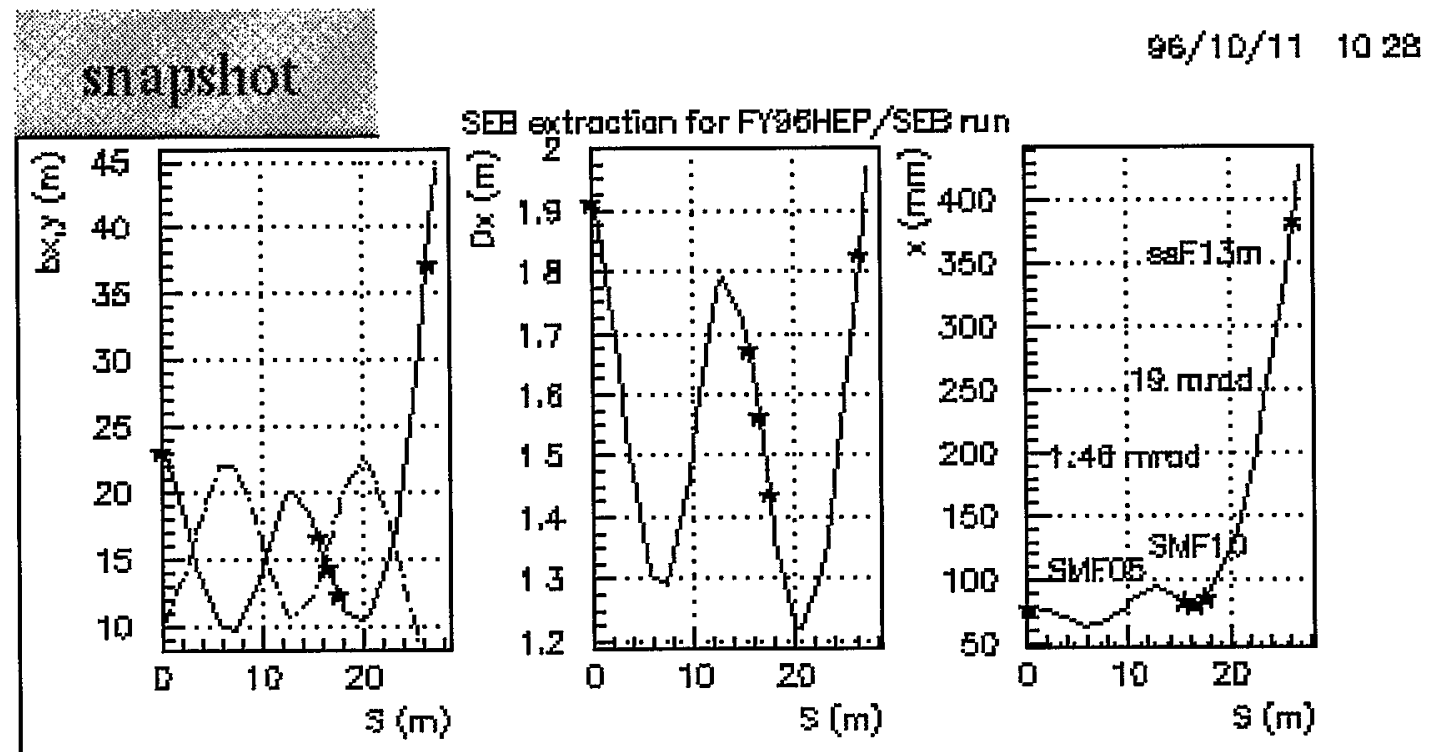

Fig. 4.5. $\beta_{h, v}, D_{X}$ and $x$ as a fuction of S starting from SMF05ds for the extracted beam. 
Table 3. FTWISS output for the extracted beam with $\theta_{\mathrm{f} 05}=1.46 \mathrm{mrad}$ and $\theta_{\mathrm{f} 10}=19.0 \mathrm{mrad}$.

LATTICE PARAMETERS FOR BEAM LINE: "SEB F05ds-to-F13md ", DELTA(P)/P = 0.000036 SYMM = F

\begin{tabular}{|c|c|c|c|c|c|c|c|c|c|c|c|c|}
\hline ELEMENT SEQ & & & $\mathrm{H}$ & O R & $\mathrm{ZO}$ & A L & & & I & VE & te & A L \\
\hline $\begin{array}{l}\text { POS. ELE } \\
\text { NO. NAME. }\end{array}$ & $\begin{array}{l}\text { I } \\
\text { I }\end{array}$ & $\begin{array}{l}\text { DIST } \\
{[\mathrm{m}]}\end{array}$ & $\begin{array}{l}\mathrm{B}_{\mathrm{h}} \\
{[\mathrm{m}]}\end{array}$ & $\begin{array}{l}\alpha_{h} \\
{[1]}\end{array}$ & $\begin{array}{c}\mu_{\mathrm{h}} \\
{[2 \pi]}\end{array}$ & $\underset{[\mathrm{mm}]}{\mathrm{x}}$ & $\begin{array}{c}x^{\prime} \\
{[.001]}\end{array}$ & $\begin{array}{l}\mathrm{D}_{\mathrm{X}} \\
{[\mathrm{m}]}\end{array}$ & $\begin{array}{ll}\mathrm{D}_{\mathrm{X}}^{\prime} & \mathrm{I} \\
{[1]} & \mathrm{I}\end{array}$ & $\begin{array}{l}\beta_{\mathrm{V}} \\
{[\mathrm{m}]}\end{array}$ & $\begin{array}{l}\alpha_{\mathrm{V}} \\
{[1]}\end{array}$ & $\begin{array}{c}\mu_{\mathrm{V}} \\
{[2 \pi]}\end{array}$ \\
\hline & 1 & & & & & & & & & & & U. \\
\hline F10us & 1 & 15.469 & 4 & & 0.171 & 83.1 & & & -0.109 & & & 0.170 \\
\hline $18 \mathrm{SN}$ & & .484 & 287 & 1.098 & 0.182 & 79.6 & & & -0.119 & & & 0.181 \\
\hline $19 \mathrm{~S}$ & 1 & 17.499 & 12.218 & 0.941 & 0.194 & 85.7 & 10.7 & & -0.128 & & & 0.191 \\
\hline $22 \mathrm{~N}$ & 1 & 20.015 & 10.419 & -0.3 & 0.232 & $124.10 ?$ & 722 & 1.226 & 0.018 & & & 0.210 \\
\hline $\mathrm{F} 12$ & 1 & 631 & & & 0.266 & 200. & 84 & & 0.128 & & & 0.230 \\
\hline 13 & 1 & 628 & & & 0.289 & 339. & & & 0.238 & & & 0.26 \\
\hline F13md & $\mathbf{1}$ & 26.390 & 37.175 & 4.439 & 295 & 30 & & 1.827 & 0.238 & & & 0.287 \\
\hline
\end{tabular}

\section{Results}

- Lets first compare our tracking results at ssF13md for the FY96 HEP/SEB with the values quoted as the nominal values used for design in the AGS SEB switchyard design report[3] and 1979 Weisberg's measurements[2]:

\begin{tabular}{|c|c|c|c|c|}
\hline & Design Report & Weisberg & FY96 MAD & \\
\hline $\begin{array}{l}\varepsilon^{* e^{2}} h_{h}(95 \%)^{\dagger} \\
\beta_{h} \\
\alpha_{h}\end{array}$ & $\begin{array}{l}31.4 \pi \\
36.68 \\
-4.565\end{array}$ & $\begin{array}{l}37.7 \pi \\
58.42 \\
-6.6\end{array}$ & $\begin{array}{c}54.0 \pi \\
37.175 \\
-4.439\end{array}$ & $\begin{array}{l}\text { mm-mrad } \\
\mathrm{m}\end{array}$ \\
\hline $\begin{array}{l}\varepsilon^{* e x t}(95 \%) \dagger \\
\beta_{V} \\
\alpha_{V}\end{array}$ & $\begin{array}{l}52.3 \pi \\
3.76 \\
1.032\end{array}$ & $\begin{array}{l}45.5 \pi \\
3.30 \\
0.87\end{array}$ & $\begin{array}{l}(90.0 \pi) \\
6.106 \\
1.156\end{array}$ & $\begin{array}{l}\mathrm{mm} \text {-mrad } \\
\mathrm{m}\end{array}$ \\
\hline Dx & & & 1.827 & $\mathrm{~m}$ \\
\hline
\end{tabular}

$\uparrow \mathrm{NB}$ : The values in ref.[2] and [3] are unnormarized $99 \%$ values at $\mathrm{p}=29 \mathrm{GeV} / \mathrm{c}$ and are converted

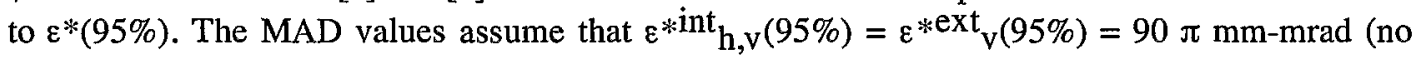
vertical blow-up).

The FY96 MAD simulation results are consistent with both the switchyard design report values and Weisberg's values except the emittance, which is much higher, merely reflectis the assumed larger internal emittance of $\varepsilon^{* \text { int }_{\mathrm{h}, \mathrm{v}}}(95 \%)=90 \pi \mathrm{mm}$-mrad for the high beam intensity of $5-6 \cdot 10^{13} \mathrm{ppp}$. It should be noted that the MAD tracking predicts that the external horizontal emittance should be $\sim 60 \%$ of the internal emittance once the momentum sweep during spill is corrected. These values should be compared with the recent measurements once they are available[7].

- Due to the chromaticity correction made in FY96 to cope with the vertical beam instability, 
-the instantaneous momentum spread ldp/pl should have increased from $0.023 \%$ to $0.057 \%$ for a total $\mathrm{ldp} / \mathrm{pl}=\sim 0.4 \%$ after debunching as it is porpotional to $1 / \xi_{\mathrm{h}}$,

-the difference between the step size for the zero and for the nominal emittance increased from 3.0 $\mathrm{mm}$ to $3.8 \mathrm{~mm}$ at ESH20us and 14.6 to $22.1 \mathrm{~mm}$ at SMF05us. The horizontal beam size could be already too large at SMF05, causing extra beam loss.

- The1.14 mm clearance at SMF05 is marginal. It could be improved if we could increase the ESH20 kick though it is better to use the ESH20 kick directly rather than using the non-linear effect to create the gap at SMF05[8].

- The overall general characteristics of the SEB extraction obtained in this study are consistent with the previous simulation studies $[2,8]$.

\section{Conclusions}

The AGS SEB extraction had been studied by the MAD tracking using the actual FY96 HEP/SEB machine setup parameters and an estimated emittance $\varepsilon^{*} \mathrm{~h}(95 \%)=90 \pi \mathrm{mm}-\mathrm{mrad}$ for the high intensity beam of $5-6 \cdot 10^{13} \mathrm{ppp}$ to predict the extracted beam parameters at $\mathrm{ssF} 13 \mathrm{md}$.

- The predicted beam parameters $(\alpha, \beta)$ at ssF13md are consistent with the switchyard design values and also with Weisberg's.

- However, the external emittance is substantially larger than the previous values simple because we use

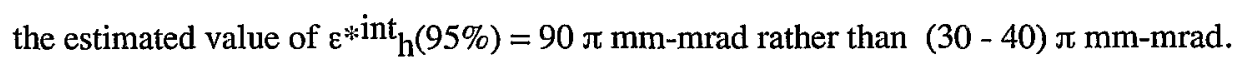

- It is crucial for us to know exactly what the internal beam emittance is. We should make every effort to measure both the horizontal and vertical beam emittance on the flattop and its intensity dependency in a controlled fashion.

- Both horizontal and vertical tracking from SMF05 to ssF23md for the extracted particles can be repeated using a full field map rather than the simple parameterization of the F13 main magnet and taking particle distribution into account [9]. 


\section{Acknowledgments}

The author would like to thank Jim Niederer for his continuous efforts on improving the BNL MAD code and developing the user-friendly interactive graphical display utility. Without his help this tracking study would be more tedious and time consuming work.

\section{References}

[1] M.Q. Barton and J. Faucre, "Improved Calculations of the Slow Extracted Beam", AADD-131 (1967). L.N. Blumberg et al, "Initial Performance of the AGS Slow External Beam", IEEE No.167, 234 (1967).

[2] H. Weisberg, "Option for Improving the Slow Extracted Beam", AGS/Tech. Note No.131 (1977), J.W. Glenn et al, "AGS Slow Extraction Improvements", IEEE NS26,No.3, 3167 (1979) H. Weisberg and J.W. Glenn, "Exploitation of Nonlinear Growth of Betatron Oscillations to Obtain Efficient Slow Extraction at the AGS", Nucl. Inst. \& Methos 169, 319(1980).

[3] L. Blumberg et. al., "The AGS Slow External Beam Switchyard”, BNL 24508R (1979)

[4] M. Tanaka, "An Overview of the Slow Extraction at the AGS", AGS/AD/Tech Note No. 334

[5] M. Tanaka, "SEB Extraction Study I", AGS/AD/Studies Report No.219. M. Tanaka and J.W. Glenn, " SEB Extraction Study II", ibid, No.222. M. Tanaka, "SEB Extraction Study III", ibid, No. 229.

[6] J. Niederer, BNL MAD Program Notes.

[7] J. Glenn and K. Brown, private communication, The FY96 SEB emittance measurements are now available in AGS/AD/Tech Note No. 445.

[8] Ch. Steinbach, "Study of a New Extraction Scheme", AGS/AD/Tech.Note No.280 (1987).

[9] A. Luccio, private communication. 